\title{
Power Problem
}

National Cancer Institute

\section{Source}

National Cancer Institute. Power Problem. NCI Thesaurus. Code C63025.

Problem associated with the energy to operate the device. 